\title{
Study of Vortex State in Permalloy Plates Using Optimized Electron Holography
}

Eduardo Ortega ${ }^{1}$, Beatriz Mora ${ }^{2}$, Carlos Monton $^{1}$, Carolina Redondo $^{2}$, Josefina Arellano ${ }^{1}$, Rafael Morales $^{3,4}$, Edgar Voelkl ${ }^{6}$, Arturo Ponce ${ }^{1,5}$

1. Department of Physics and Astronomy, University of Texas at San Antonio, San Antonio, Texas, United States

2. Department of Physical Chemistry, University of the Basque Country, Lejona, Vizcaya, Spain

3. BCMaterials, University of the Basque Country, Lejona, Vizcaya, Spain

4. IKERBASQUE, Basque Foundation for Science, Bilbao, Spain

5. National Institute for Astrophysics, Optics and Electronics, Santa Maria Tonantzintla, Puebla, Mexico.

6. HoloWerk LLC, Germantown, Maryland, United States

A key interest in the reduction of dimensions in ferromagnetic materials is the generation of flux-closure states called vortex states. This magnetic vortex state typically develops in low aspect ratio nanostructures due to the increase magnetostatic self-energy in contrast with their continuous thin film or bulk counterparts [1]. During the past decade, special emphasis was devoted to the study of induced magnetostatic interactions using nanodisks and plates [2]. These magnetic nanostructures (MNSs) easily respond to an external magnetic field and develops long-range magnetic interactions. Interestingly, they can be bio-functionalized with cancer biomarkers, proteins, or DNA, allowing for the development of biomedical sensors with improved sensibility [3]. It is expected that this emerging technology may produce a remarkable impact in early stage detection of grave and lethal diseases. Notwithstanding, for this technique to be successful, the MNS must remain fully demagnetized during remanence to minimize problems with agglomeration and undesirable migration. To fulfil this restriction, the vortex state of the MNSs can be tuned to have a zero-sum magnetization during remanence by engineering the shape and composition of the MNSs [4]. In this work, we present the micromagnetic analysis of individual squared plates of permalloy $\left(\mathrm{Py}, \mathrm{Ni}_{80} \mathrm{Fe}_{20}\right)$ in the vortex state using electron holography $(\mathrm{EH})$.

EH provides the most direct and reliable access to the image phase but its requirements are strict and the coherence of the electron source remains a significant limitation. Electron holography has been performed in a JEOL ARM 200F, operated at $200 \mathrm{keV}$. Holograms have been reconstructed live and in real time to study the magnetic behavior of the samples above described. The quality of the holograms has been optimized as a function of exposure time $\left(\mathrm{t}_{\exp }\right)$ and condenser setting using an algorithm in HoloWorks able to determine the optimum acquisition condition as shown in Fig. 1. In literature, interference fringe contrast is often referred as the key parameter for hologram quality. However, a hologram with $100 \%$ fringe contrast, $\mu$, but no electrons is just as bad as a hologram with infinite number of electrons $(\mathrm{N} \rightarrow \infty)$ and $\mu=0 \%$. The optimum fringe contrast is proportional to the expression for hologram quality $\mathrm{Q} \sim \mu$ $\operatorname{sqrt}(\mathrm{N})$, defined by the brightness of the electron gun. Another parameter needing consideration is the drift of the interference fringes over time, creating an upper limit to texp. Less known is the detail that there also is a lower exposure time limitation due to the beam blanker of the microscope [5]. Typically, as the beam returns from its deflection it slows until it comes to rest. That delay timeframe with the illumination in movement is critical for EH as the biprism is far out of focus and thus strongly affected by beam movements. All these parameters can be tracked live as shown in Fig. 1. The teal colored and filled line plot tracks the interference fringe contrast, while the green line plot tracks the hologram quality (proportional to the standard deviation in the phase image). The track for the fringe drift is suppressed here for simplicity. The exposure time $t_{\exp }$ starts on the left at $0.125 \mathrm{~s}$, increases to $4 \mathrm{~s}$ and returns to $0.125 \mathrm{~s}$ 
in steps of a factor multiple of 2. The Q-factor therefore should change in increments of sqrt(2), i.e. in decreasing order: $[14.8,10.5,7.4,5.3,3.7,2.6,1.85]$, whereas the measured values are $[14.8,10.2,6.6$, $3.8,2.4,1.4]$ indicating a significant drop below the expected values for $Q$ due to the shutter speed of the beam deflector above the sample. These values limit our hologram acquisition to a range of $1-4 \mathrm{~s}$ in the microscope used. Consecutive single holograms are registered in image stacks yielding high phase sensitivity on a regular basis. Examples of holographic imaging of magnetic fields is shown in Fig. 2, in which we have registered electron holograms over a field of view of $1 \mu \mathrm{m}^{2}$ and a fringe contrast of $\mu \approx$ $25 \%$ under free lens conditions. The holograms were recorded in a CCD camera and processed using HoloWorks to extract magnetic induction and magnetic contour images (both live and off-line) as shown in Fig. 2. Interference fringes in were used. To perform a reliable magnetic analysis, the magnetic phase was separated by flipping the single MNS [6].

References:

[1] P. Vavassori et al, Appl. Phys. Lett. 86 (2005), p. 072507.

[2] M. Schneider, H. Hoffmann, and J. Zweck, Appl. Phys. Lett. 77 (2000), p. 2909.

[3] Vigneshwaran Mani, et al, Expert Opin Med Diagn. 5 (2011), p. 381.

[4] B. Mora, et al. ACS Appl. Mater. Interfaces, DOI: 10.1021/acsami.7b16779.

[5] E. Voelkl, D. Tang, Ultramicroscopy 110 (2010), p. 447.

[6] The authors acknowledge funding from MINECO FIS2016-76058 (AEI/FEDER, UE), the EU grant H2020-MSCA-RISE-2016-734801 and Department of Defense \#64756-RT- REP.

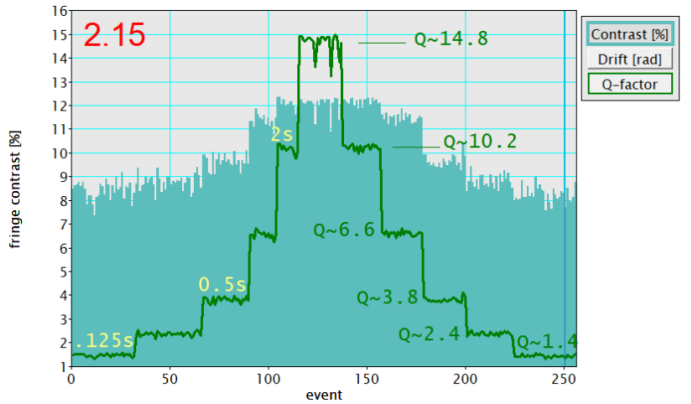

Figure 1. Hologram quality $Q$ and fringe contrast $\mu$ as a function of texp ranging from $0.125 \mathrm{~s}$ to $4 \mathrm{~s}$ and back to $0.125 \mathrm{~s}$ in a way to track hologram quality.

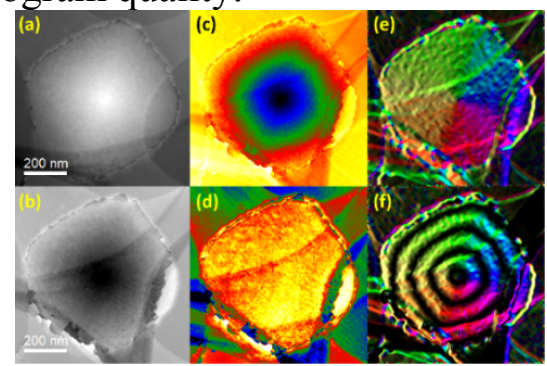

Figure 2. EH phase analysis of a single Py square. (a) Unwrapped phase of the Py obtained from the complex image. (b) Same as (a) but after flipping the sample manually. (c) Half the subtraction of (a) and (b). (d) Half the addition of (a) and (b). (e) Magnetic induction image obtained from the magnetic contribution at (c). (f) Magnetic contour image showing the vortex state. 